\title{
ABCD Renal Association Abstracts 2019
}

\author{
The 4th joint meeting of the $A B C D$ \& The Renal Association took place at the NEC in \\ Birmingham on 13th February 2019. The top three abstracts are included here and the \\ remaining can be found online at www.bjd-abcd.com
}

\author{
Intensive medical therapy is associated with increased urinary \\ excretion of neutrophil gelatinase-associated lipocalin (NGAL) \\ in experimental diabetic nephropathy \\ Chuah YHD, ${ }^{1,2}$ Hutter $M^{2}$ Abdelaal $M^{2,3}$ le Roux $C W^{2}$ \\ Docherty NG1,2 \\ UCD School of Medicine, University College Dublin, Belfield, Dublin 4, Ireland \\ 2 UCD Diabetes Complication Research Centre, School of Medicine, Conway Institute, \\ University College Dublin, Belfield, Dublin 4, Ireland \\ 3 Plastic Surgery Department, Assiut University Hospital, Assiut, Egypt
}

Elevation of urinary NGAL is primarily associated with impaired proximal tubular reabsorption and distal nephron injury. This study examined the effect of intensive medical therapy (IMT) on urinary excretion of NGAL in the Zucker Diabetic Sprague-Dawley (ZDSD) rat model of diabetic nephropathy (DN).

Adult male ZDSD rats of 26 weeks of age were allocated to undergo IMT $(n=8)$ and compared at follow-up with untreated disease control rats (DC, n=9). IMT consisted of weight-adjusted dosage of metformin, ramipril, rosuvastatin, fenofibrate and liraglutide with $20 \%$ weight loss of total body weight achieved by food restriction. Longitudinal assessment of plasma glucose levels and hourly excretion rates of urinary albumin and NGAL were conducted across baseline and post-intervention periods.

Baseline plasma glucose levels showed a stronger correlation with urinary NGAL excretion $(\rho=0.71, p<0.001)$ compared with urinary albumin excretion, the standard clinical indicator of DN $(\rho=0.47$, $\mathrm{p}=0.001$ ). At 4 weeks post-intervention, a 7.8-fold increase in the median (IQR) urinary albumin excretion was observed in DC (109.1 (261.3) $\mu \mathrm{g} /$ hour vs. 849.4 (5727.0) $\mu \mathrm{g} / \mathrm{hour}, \mathrm{p}=0.012$ ) and a 2.1fold reduction was observed in IMT (97.9 (67.4) $\mu \mathrm{g} / \mathrm{hour}$ vs. 47.5 (28.6) $\mu \mathrm{g} /$ hour, $\mathrm{p}=0.008$ ). However, a significant increase was observed in the median (IQR) urinary NGAL excretion in both groups: 2.8-fold in DC (122.4 (91.0) ng/hour vs. 344.6 (604.5) ng/hour, $\mathrm{p}=0.012$ ) and 2.9-fold in IMT (106.6 (57.9) ng/hour vs. 310.2 (110.3) ng/hour, $\mathrm{p}=0.008$ ).

IMT significantly reduced urinary albumin excretion but paradoxically elevated urinary excretion of NGAL. This suggests an association between elements of the IMT regimen and increased urinary excretion of NGAL from renal tubular injury or pre-renal origin.

Evaluation of $\mathrm{HbA}_{1 \mathrm{c}}$ in a primary care cohort with diabetes and chronic kidney disease

Currie A, Moore-Haines K, Sullivan K, Solomon A, Hardy D, Winocour $\mathrm{PH}$

ENHIDE, Welwyn Garden City and Centre for Health Service and Clinical Research, University of Hertfordshire

Glycaemic management in diabetes (DM) and chronic kidney disease (CKD) should be individualised, given the limitations of $\mathrm{HbA}_{1 \mathrm{c}}$ measurement and increased hypoglycaemic risk. The ENHIDE renal DM
Telehealth project reviewed 2,356 adults with DM and CKD from primary care diabetes registers. $\mathrm{HbA}_{1 \mathrm{c}}$ was populated in 2,325 and values varied from 23 to $148 \mathrm{mmol} / \mathrm{mol}$ (median 53), and 1,421 (61\%) had $\mathrm{HbA}_{1 \mathrm{c}}$ values at or below $58 \mathrm{mmol} / \mathrm{mol}$. In comparison with those with higher $\mathrm{HbA}_{1 \mathrm{c}}$, they had comparable haemoglobin levels (127 vs. $128 \mathrm{~g} / \mathrm{dL}$ ) and those with most significant CKD (estimated glomerular filtration rate (eGFR) $<45$ and albumin to creatinine ratio $(A C R)>10, n=273$ ) had comparable $\mathrm{HbA}_{1 \mathrm{c}}$ to the larger residual group $(n=2,052)$, with the exception of those with albuminuria $(A C R>10)$ with eGFR $>60$ whose $\mathrm{HbA}_{1}$ c levels were higher $(62$ vs. those with reduced eGFR 55-57 mmol/mol $(p<0.0005)$ ). Anaemia $(\mathrm{Hb}<110 \mathrm{~g} / \mathrm{L})$ was evident in $15 \%$ of cases, with an additional $16 \%$ treated for anaemia with $\mathrm{Hb}>110 \mathrm{~g} / \mathrm{L}$. $\mathrm{HbA}_{1 \mathrm{c}}$ values over 2 years were recorded as stable in 633, variable in 346, rising in 234 and improved in 172 cases. Overall, 690 were on insulin (including 114 also on sulfonylureas) and 458 on sulfonylureas, with the vast majority on diet alone or other diabetes therapies. Hypoglycaemia risk was identified in 174 individuals on insulin/and or sulfonylureas whose $\mathrm{HbA}_{1 \mathrm{c}}$ was recorded as $<50 \mathrm{mmol} / \mathrm{mol}$.

These data allow targeting for modification of hypoglycaemic therapy and where additional measures of glycaemia are necessary in anaemia.

All on board for the mobile diabetes clinic: a person-centred holistic approach to caring for patients with diabetes in the haemodialysis unit

Allen $T_{1}{ }^{1}$ Cummins $L,{ }^{2}$ Fogarty $D,{ }^{2}$ Gilleece $M,{ }^{2}$ Jennings $S,{ }^{2}$ Johnston PC, ${ }^{1}$ Madden J, ${ }^{3}$ Magee $K{ }^{2}{ }^{2}$ Nesbitt $P,{ }^{1}$ Nugent AG, ${ }^{1}$ Peto $T,{ }^{4,5}$ Roberts $B,{ }^{6}$ Wallace $H J,^{1}$ Wallace $I R^{1}$

Department of Endocrinology and Diabetes, Belfast City Hospital, Belfast Health and Social Care Trust, Belfast

2 Regional Nephrology Service, Belfast City Hospital, Belfast Health and Social Care Trust, Belfast

3 Podiatry Service, Belfast City Hospital, Belfast Health and Social Care Trust, Belfast ${ }^{4}$ Northern Ireland Diabetic Eye Screening Programme, Public Health Agency, Belfast

5 Centre for Public Health, Queen's University Belfast, Belfast

6 Department of Clinical Biochemistry, Belfast Health and Social Care Trust, Belfast

Patients with diabetes receiving haemodialysis are frail with multiple co-morbidities. The standard model of care was not meeting the needs of this patient cohort. In response and in collaboration with patients, we have developed a multidisciplinary mobile clinic bringing care to the bedside in the dialysis unit. We describe our model of care and the results from one year of follow-up.

Standard diabetes outpatient clinic appointments are replaced by a consultant-delivered diabetes assessment in the dialysis unit with diabetes specialist nurse and dietitian input. Dialysis nurses perform monthly foot assessments with rapid access to podiatry input. The retinal screening service brings the screening camera to the dialysis unit twice a year. 
Our dialysis unit provides maintenance haemodialysis to $182 \mathrm{pa}-$ tients. 35\% $(n=63)$ have diabetes. 90.2\% $(n=46)$ had type 2 diabetes. Mean $\mathrm{HbA}_{1 \mathrm{c}}$ and duration of diabetes were $69.0 \mathrm{mmol} / \mathrm{mol}$ and 19.7 years, respectively. $66.7 \%$ were treated with insulin. $51 \%$ had diabetic nephropathy $(n=26) .88 .2 \%$ required assistance with transport and $72.5 \%$ used a mobility aid. Mortality during this period was $12.7 \%(n=8) .37 .3 \%$ had not attended retinal screening in the previous year. Of these, one in four had sight-threatening retinopathy requiring urgent ophthalmology intervention. $21.6 \%$ had active foot disease and were reviewed by podiatry onsite.

The standard model of care does not fit the needs of this cohort. Our innovative mobile clinic detected a significant number of complications requiring urgent treatment and has resulted in all patients $(100 \%)$ receiving their nine key care processes (NICE).

\section{Hospital hypoglycaemia is a more common event in renal compared with other wards \\ Zac-Varghese S, Sisley M, Peprah D, Mathew S, Hardy D, Hodgkins S, Baldry N, Stanisstreet D, George S, Winocour P \\ East and North Herts Institute of Diabetes and Endocrinology (ENHIDE) \\ Sisley M and Peprah D are UCL final year medical students}

In July 2018 the East and North Herts NHS Trust introduced linked glucose meters to monitor occurrences of hypoglycaemia amongst hospital inpatients. Data collection and analysis on 32 days between July and October demonstrated a daily occurrence of 8-19 episodes of hypoglycaemia (with or without diabetes) (average 11 (mode)). There was a marked variation across all wards with a higher incidence of hypoglycaemia on the renal and dialysis wards compared with other wards. Out of a total of 425 episodes, $20 \%$ of these occurred on the renal wards. The wards with the lowest incidences of hypoglycaemia were respiratory (1\%), gastroenterology and stroke. We analysed data during a 1-week period using a standard audit form based on JBDS hypoglycaemia guidance. Universally, the guidance was not followed. 19 cases were audited in greater depth and in only one out of 19 was a normal blood glucose recorded following the event. In most cases the treatment for hypoglycaemia was not recorded and invariably there was no discussion or consideration of the possible cause of hypoglycaemia. This initial analysis of data demonstrates a worrying prevalence of hypoglycaemia and poor treatment and lack of adherence to guidance.

We are currently evaluating knowledge on hypoglycaemia to determine the reasons for lack of adherence to guidelines, focusing on the inpatient renal staff. Based on this survey, we intend to carry out an innovative staff education programme and repeat the audit of data.

Discordant trajectories in urine albumin and NGAL excretion with addition of gastric bypass surgery to best medical therapy for type 2 diabetic kidney disease Martin WP, Islam MN, Aboud CM, da Costa Silva AC, da Silveira LP, Petry TB, Cohen RV, Griffin MD, le Roux CW, Docherty NG

Diabetes Complications Research Centre, Conway Institute of Biomolecular and Biomedical Research, School of Medicine, University College Dublin, Dublin, Ireland Regenerative Medicine Institute (REMEDI), School of Medicine, College of Medicine, Nursing and Health Sciences, National University of Ireland, Galway, Ireland

The Centre for Obesity and Diabetes, Oswaldo Cruz German Hospital, São Paulo, Brazil
Introduction: Metabolic surgery reduces albuminuria in type 2 diabetes mellitus (T2DM), but its renal tubular impact is not well characterised. Urinary neutrophil gelatinase-associated lipocalin (NGAL) identifies renal tubular injury.

Methods: Microvascular Outcomes after Metabolic Surgery (MOMS) is a clinical trial (NCT01821508) in which 100 individuals with T2DM, microalbuminuria and BMI 30.0-34.9 kg/m² have been randomised to combined gastric bypass surgery with medicine (CSM, $n=51$ ) or medical therapy alone (MTA, $n=49)$. NGAL concentrations were measured in spot urine samples of MOMS participants at baseline and 6 months by ELISA and adjusted for urinary creatinine. Data analysis was performed in $\mathrm{R}$.

Results: N (\%) female, n (\%) Caucasian, BMl, $\mathrm{HbA}_{1 c}$, serum creatinine, urine albumin:creatinine ratio $(A C R)$ and urine NGAL:creatinine ratio for the CSM vs. MTA arms at baseline were: 23 (45.1\%) vs. 22 $(44.9 \%)(p=0.98) ; 46(90.2 \%)$ vs. $34(69.4 \%)(p=0.18) ; 32.52 .0$ vs. $32.82 .2 \mathrm{~kg} / \mathrm{m} 2$ ( $p=0.47) ; 72.620 .3$ vs. $74.221 .6 \mathrm{mmol} / \mathrm{mol}(\mathrm{p}=0.53) ;$ 71.619 .2 vs. $74.423 .6 \mu \mathrm{mol} / \mathrm{L}(p=0.53) ; 8.1$ (9.9) vs. $8.2(13.1)$ $\mathrm{mg} / \mathrm{mmol}(\mathrm{p}=0.53)$; and 582.5 (1529.5) vs. 831.0 (1044.6) $\mathrm{ng} / \mathrm{mmol}$ $(p=0.36)$. Baseline vs. month 6 urine ACR and urine NGAL:creatinine in the CSM arm were: 8.1 (9.9) vs. $2.0(2.1) \mathrm{mg} / \mathrm{mmol}(p<0.001)$ and 582.5 (1529.5) vs. 805.6 (1372.7) ng/mmol ( $p=0.23)$. Corresponding values in the MTA arm were: 8.2 (13.1) vs. 2.7 (7.3) $\mathrm{mg} / \mathrm{mmol}(p<0.001)$ and $831.0(1044.6)$ vs. 754.8 (2467.2) $\mathrm{ng} / \mathrm{mmol}(\mathrm{p}=0.16)$. Percentage delta change in urine ACR and urine NGAL:creatinine from baseline to month 6 in the CSM vs. MTA arms were: $-79.5(18.1) \%$ vs. $-68.1(51.0) \%(p=0.075)$ and +102.6 (332.5)\% vs. $-44.6(141.2) \%(p=0.047)$.

Conclusion: Urine NGAL excretion is increased at 6 months postCSM but not MTA in T2DM, despite similar reductions in albuminuria.

ENHIDE Telehealth primary care support of adults with diabetes and chronic kidney disease: a pilot study Winocour PH, Moore-Haines K, Solomon A, Currie A, Hardy D ENHIDE, QE2 Hospital, Howlands, Welwyn Garden City, Herts

Diabetes (DM) and chronic kidney disease commonly co-occur and should be considered a multi-morbid complex disorder that requires a holistic approach, recognising the impact of cardiovascular disease, retinopathy, bone health, anaemia, increased hypoglycaemia risk and foot and eye complications of diabetes. National and international audits have demonstrated failures in achieving standard blood pressure, lipid and glycaemic targets. A pilot project involving 20 general practices was initiated in 2016 to examine the feasibility of comprehensive big data extraction and analysis enabling individualised patient care carried out through Telehealth case-based discussion and primary care up-skilling, along with provision of patient information to enable self-management of acute illness and foot health. All patients with estimated glomerular filtration rate (eGFR) $<60$ and/or urine albumin:creatinine ratios $>10$ were identified. To date, almost 2,400 cases have been evaluated of whom $45 \%$ were aged $<75$, reflecting $24 \%$ of those on DM practice registers. On average, over $80 \%$ were solely under primary care. A significant unmet need was identified: $17 \%$ required referral to specialist renal units through significant deterioration of renal function, $10 \%$ for enhanced diabetes care, $26 \%$ of cases required changes to antihypertensive therapy, changes in lipid-lowering therapy in 37\%, changes to glycaemic medication in $26 \%$ and antiplatelet therapy recommended in $11 \%$. In addition, $26 \%$ had a high risk of active foot 
problems requiring advice and $32 \%$ (where eGFR $<45$ ) required assessment of metabolic bone health. Anaemia was present in 13\% - more so in those aged $>75$. Primary care valued the Telehealth process.

The impact of age and body mass index on biomedical measures in a primary care population with diabetes and chronic kidney disease

Moore-Haines K, Hardy D, Sullivan K, Blackwell L, Solomon A, Currie A, Winocour PH

ENHIDE, Welwyn Garden City and Centre for Health Service and Clinical Research, University of Hertfordshire

There is published evidence that obesity in the elderly may be associated with better health outcomes. In our Telehealth project evaluating the feasibility of supporting primary care in the management of people with type 2 diabetes (DM) and chronic kidney disease (CKD), we identified 2,356 such individuals from 17 participating practices, representing $23 \%$ of those on the practice diabetes registers. 1,349 (57\%) had estimated glomerular filtration rate (eGFR) $<60$ and normal albumin:creatinine ratios (ACR) or no recent ACR information, $493(21 \%)$ had eGFR $<60$ and raised ACR $(>10)$ and $514(22 \%)$ had eGFR $>60$ and ACR $>10$. This current analysis examined the impact of age ( $<$ or $>75$ years) and body mass index (BMI, $<$ or $>30 \mathrm{~kg} / \mathrm{m}^{2}$ ) on cardiometabolic measures.

Those aged $>75$, regardless of BMI, had significantly lower eGFR (48 vs. $56 \mathrm{~mL} / \mathrm{min}$ ) and haemoglobin (124-125 vs. $132 \mathrm{~g} / \mathrm{dL}$ ), ACR (3338 vs. $46-65)$, diastolic blood pressure (73-74 vs. $75-76 \mathrm{mmHg})$, $\mathrm{HbA}_{1 \mathrm{c}}$ ( 53-55 vs. 62-63 mmol/mol), cholesterol (4.2-4.3 vs. 4.5$4.6 \mathrm{mmol} / \mathrm{L})($ all $\mathrm{p}<0.0005)$. By contrast, obese patients $(\mathrm{BMI}>30)$ had somewhat higher levels of systolic blood pressure (135-137 vs. $134-135 \mathrm{mmHg}$ ) regardless of age $(p=0.027)$.

These data suggest that older obese DM patients with CKD in the community do not have a lower adverse cardiometabolic risk than younger obese or non-obese patients regardless of age. Elderly DM patients with CKD have greater reductions in eGFR and haemoglobin but apparently less adverse values of other cardiometabolic measures.

Renal diabetes champions: improving the quality of care for people with diabetes on chronic haemodialysis Mohamed M, Young P, Patterson P, McWhan L, Davidson K, Kelly $M$

All NHS Dumfries and Galloway, no affiliations

In Dumfries and Galloway we have 20 adults with diabetes on haemodialysis. Many start dialysis with multiple complications. They are at high risk of hypoglycaemia, foot ulcers, infections and amputation. After attending ABCD:RA 2017 we appointed diabetes renal champions from renal, diabetes and podiatry teams. Our aim was to implement UK ABCD-RA guideline recommendations about organisation of care through multidisciplinary team (MDT) work, anticipatory and preventative care. We identified several areas to improve our local service delivery: (1) monitoring and documenting pre- and post-treatment blood glucose for each dialysis session; (2) training nurses to manage hypos on dialysis; (3) design, training and implementing regular foot checks and routine use of heel protectors on dialysis; (4) regular meetings to discuss patients in need of review and change of treatment.
On 14 June 2018 the records of 19 patients were reviewed for a total of 870 sessions. Pre-dialysis glucose was recorded for $97 \%$ of sessions and post-dialysis glucose was documented in $65 \%$ of the sessions. $100 \%$ of patients had foot screening and weekly foot checks were done on $60 \%$ of eligible sessions.

We re-evaluated our performance on 17 January 2019. The records of 12 patients were reviewed for a total of 383 sessions. Pre-dialysis glucose was recorded for $98 \%$ of sessions and post-dialysis glucose was documented in $83 \%$ of the sessions. $100 \%$ of patients had foot screening and weekly foot checks occurred in $87 \%$ of eligible sessions.

Staff are now confident in managing hypoglycaemia and referring appropriately to the diabetes team and podiatry service. Using an MDT approach, a small motivated team can bring positive changes in practice. Best standards of care can be implemented by cascading of training to all stakeholders to ensure sustainability.

Improving inpatient diabetic management in haemodialysis patients: a quality improvement project Smith A, Swift O, Morlidge C, Findlay A

Department of Renal Medicine, Lister Hospital, East and North Hertfordshire NHS Trust

The prevalence of diabetes is set to increase to $4.4 \%$ from $2.8 \%$ by 2030. Some of the most vulnerable and severely affected patients are those with end-stage renal failure requiring haemodialysis. Until recently, sufficient national guidance to support the management of this population was lacking. In 2016 the Joint British Diabetes Society and Renal Association produced guidelines covering the extensive and distinct needs of these patients. Using these guidelines as a framework, we reviewed the management of inpatient diabetic haemodialysis patients at a district general hospital with a large tertiary renal unit.

Baseline data of 12 inpatients revealed issues with communication of hypoglycaemic episodes between nurses and doctors, use of separate paperwork to document pre- and post-dialysis capillary blood glucose (CBG) measurements, and discrepancies in the management of pre-dialysis CBGs of $<7 \mathrm{mmol} / \mathrm{L}$.

Primary interventions included education, a review of where CBG measurements are best recorded, strategies to manage pre-dialysis CBG measurements and improved notification of hypoglycaemic events.

Re-audit 3 months later identified seven inpatients and demonstrated a reduction in hypoglycaemic events from $50 \%$ to $38 \%$, an uplift in prescription of bespoke insulin regimens to include dose reduction on dialysis days (0\% to $25 \%$ ), and marked improvement in the appropriate treatment of hypoglycaemia (0\% to $100 \%)$. Inspection rates for diabetic foot ulcers remained poor across both cycles $(33 \%$, $25 \%$ ). Unfortunately, these observed improvements, particularly in the rate of hypoglycaemic events, were not sustained in our most recent quality improvement (QI) cycle 6 months later.

In summary, using QI methodology we have been able to demonstrate that simple interventions can translate into measurable improvements for this complex patient group. However, we suggest that these strategies are best sustained through a systemic cultural change. As such, we are currently looking to establish a dynamic Renal/Diabetes MDT and are developing materials to improve patient awareness and self-management. We hope to report on the outcome of these interventions in the near future. 
Improved diabetes foot screening and sign posting to referral pathways on the haemodialysis unit

\section{Murphy A, Guy J, Ward A, Saeed M, Wilson I}

University Hospital Birmingham

College of Medical and Dental Sciences, University of Birmingham

Background: NICE states that patients with diabetes on haemodialysis are at high risk of foot disease, with a tenfold increase in amputation risk over the general diabetes population. Newly published JBDS guidelines allow review against national standards.

Aims: To audit current foot care standards prior to implementation of JBDS recommendations.

Method: The audit was undertaken in a community haemodialysis unit in the West Midlands. Data were collected via a questionnaire completed by the patient and dialysis nurse, including demographics and risk factor profile, foot ulceration history, foot care provision and foot education recall.

Results: $63(52 \%)$ of 121 patients had diabetes. Of those, $81 \%$ had diabetes for $<10$ years and $58 \%$ had been on dialysis for $>5$ years. Fifteen $(23 \%)$ had a history of foot disease including five minor and one major amputation and six active ulcers. $54 \%$ of patients with diabetes, $47 \%$ with any ulcer history and $33 \%$ with active ulceration had no regular podiatry review. $90 \%$ of patients with diabetes, $87 \%$ with a history of ulceration and $100 \%$ with active ulceration reported foot care monitoring. All those with active ulceration, 53\% with past ulceration and $47 \%$ of all diabetes patients reported receiving foot care advice in the past year.

Conclusions: (1) Over half of these high-risk patients received no regular podiatry review with some receiving no regular foot care of any description. (2) The majority recalled no provision of foot care advice. (3) All active foot ulcers had care, but not specific foot protection team involvement.

Recommendations: (1) Improved podiatry access. (2) Creation of local dialysis foot guidance including weekly foot inspections and improved pathway signposting.

A review of the QEHB Renal-Endocrine Complex Bone Disease Advice Service

Ullah R, Gittoes NJ, Eddington H

Queen Elizabeth Hospital, University Hospitals Birmingham

2 University of Birmingham

Introduction: Chronic kidney disease-mineral bone disorder (CKD$M B D$ ) includes a wide range of bone conditions. These conditions are difficult to manage and this created the need for the RenalEndocrine Complex Bone Advice Service. Using data collected, we have explored associations in possible biomarkers and performed a service review, comparing expert opinions from both specialties to identify variations in practice.

Methods: 97 patients have been reviewed in the service. All data have been reviewed and 88 included in the analysis (exclusions due to missing data). When comparing biochemical markers we used Spearman's rank correlation and when comparing these against fractures we used the Mann-Whitney test. The survey results are discussed subjectively.

Results: Significant associations were shown between alkaline phosphatase and the bone turnover markers C-terminal telopeptide $(p=0.01)$ and procollagen-type1 propeptide $(p=0.02)$. Low 25 hydroxyvitamin $D$ levels and high parathyroid hormone were associated with an increased risk of fragility fractures. A survey of consultant nephrologists and endocrinologists had 15 responses for analysis. 60\% named relevant side effects for bisphosphonates and $33 \%$ for denosumab. Scenarios also showed variation in responses and some recommended contraindicated medication. Feedback on how the service could be developed was also gained.

Conclusion: The biochemical markers need further study in a less biased population but do show that these have a role in assessing patients with renal disease. The survey suggests that joint specialist input is useful in guiding clinical management in complex bone disease and CKD and we now need to focus on how we can improve the delivery of this.

Acute kidney injury in medical admissions: results of audits of prevalence and of staff knowledge

Bogusz $P_{,}^{1}$ Moran $C_{1}^{2}$ Fogarty $J_{1}^{1}$ Wallace $H J,{ }^{1}$ lqbal N,2

Johnston $P C,{ }^{1,4}$ Connolly $G^{3}$ Wallace IR, ${ }^{1,4}$ Nugent $A G^{1}$

1 Department of Endocrinology and Diabetes, Belfast City Hospital, Belfast Health and Social Care Trust, Belfast

2 Department of Endocrinology and Diabetes, Mater Hospital, Belfast Health and Social Care Trust, Belfast

3 Department of Clinical Biochemistry, Belfast Health and Social Care Trust, Belfast

4 Acute Medicine Unit, Royal Victoria Hospital, Belfast Health and Social Care Trust, Belfast

Acute kidney injury (AKI) is common in acute medical admissions. AKI is associated with increased mortality and prolonged length of stay. Advice to hold nephrotoxic medications at times of illness may not be getting through to patients. We retrospectively reviewed all 238 medical admissions in a 1-week period (20-27 November 2017). AKI was present in 61 (26\%). 30 of the 61 patients (49\%) were taking nephrotoxic medications and only one (2\%) stopped these agents. On further analysis we separated patients with AKI into three cohorts: patients with standard dispensing ( $n=33,54 \%)$, those with weekly blister-packs ( $n=20,33 \%)$ and nursing home residents $(n=8,13 \%) .11$ of 20 (55\%) with blister-packs were prescribed nephrotoxic medications and were stopped in none. Nephrotoxic medications were prescribed in all eight nursing home residents and were held in none.

Secondly, we performed an electronic questionnaire of staff knowledge, completed by 34 medical staff of all grades. $82 \%$ of medical staff were aware of nephrotoxic sick day rules. $29 \%$ stated they advised patients of these rules at clinic appointments. $92 \%$ of medical staff would like a kidney safety card.

Thirdly, we assessed patient knowledge by written questionnaire. 51 were returned. $78 \%$ were not aware of nephrotoxic sick day rules. $84 \%$ would like a kidney safety card.

We demonstrate a high prevalence of AKI, low patient knowledge of the potential for AKI due to their medications, and a low level of staff education of patients with both patients and medical staff wanting a kidney safety card, which we are presently developing.

Cumulative fluid balance is a risk factor for AKI development and progression

Zhang $J,{ }^{1,4}$ Seylanova $N^{2,4}$ Crichton $S,{ }^{3}$ Dixon $A,{ }^{4}$

Ostermann $\mathrm{M}^{4}$

1 Department of Critical Care, Zhongnan Hospital of Wuhan University, Wuhan, China

2 Department of Pathophysiology, Sechenov First Moscow State Medical University, Moscow, Russia

3 Medical Research Council Clinical Trials Unit, University College London, London, UK

4 Department of Critical Care, Guy's and St Thomas' NHS Foundation Trust, London, UK 
Background: Acute kidney injury (AKI) is known to be a risk factor for mortality. Our aim was to identify risk factors for the development and progression of AKI in critically ill patients.

Methods: We analysed 2,525 patients without AKI or end-stage renal disease who were admitted to ICU from January 2014 to December 2016 and explored the risk factors for development and non-recovery of $\mathrm{AKI}$.

Results: The incidence of AKI in 7 days was 33\%. Multivariate analysis showed that body mass index, SOFA score, chronic kidney disease (CKD) and fluid balance were independent risk factors for the development of AKI. Among patients who developed AKI in ICU, 69\% had full renal recovery, $8 \%$ partial recovery and $23 \%$ had no recovery of renal function. Survival analysis showed that AKI patients without renal recovery had significantly higher hospital mortality $(60 \%)$ than other groups. Independent risk factors for renal non-recovery were CKD, respiratory, mechanical ventilation, diuretic and extreme fluid balance before/after AKI day.

Conclusions: $\mathrm{AKI}$ is common and mortality is highest in those who do not recover renal function. Cumulative fluid balance impacts the chances of AKI development and progression.

\section{Are we managing our diabetic microalbuminuria patients optimally?}

\section{Subbiah K, Sivappriyan S}

Maidstone and Tunbridge Wells NHS Trust

Diabetic nephropathy is a major cause of end-stage renal disease worldwide. It is facilitated by poor long-term glycaemic control, hypertension, dyslipidaemia and cigarette smoking. Microalbuminuria (urine ACR between 3 and $29.9 \mathrm{mg} / \mathrm{mmol}$ ) is the earliest indicator of kidney damage and detecting microalbuminuria offers the chance of early intervention.

In our study at Maidstone Hospital we analysed the data of 30 clinic patients with diabetes and persistent microalbuminuria (type 1, $\mathrm{n}=13$; type $2, \mathrm{n}=17$ ) over a 6 -month period between January and June 2018 to review our performance with regard to risk factor management. The average patient age was 52 years and the duration of diabetes was between 2 and 40 years. Of the 20 patients who had an estimated glomerular filtration rate (eGFR) of $\geq 90$ $\mathrm{mL} / \mathrm{min}$, only $25 \%$ were labelled as having chronic kidney disease stage $1.43 .3 \%$ of patients had an average blood pressure above target. Only $23.3 \%$ of patients were on ACE inhibitor or ARB therapy with no identifiable contraindications to treatment in the remaining patients. $86.6 \%$ had a $\mathrm{HbA}_{1 \mathrm{c}}>53 \mathrm{mmol} / \mathrm{mol}$. Of the 11 patients with type 2 diabetes and eGFR $\geq 60 \mathrm{~mL} / \mathrm{min}, 18.1 \%$ were on SGLT2 inhibitors. $11.7 \%$ of all type 2 diabetes patients were on GLP-1 receptor agonists. $56.6 \%$ of patients had a low density lipoprotein $\geq 2.6 \mathrm{mmol} / \mathrm{L}$ and, of these patients, only $35.2 \%$ were on statin therapy. Overall, $40 \%$ of patients were on statin therapy. $23.3 \%$ of the patients smoked cigarettes and none were offered cessation counselling. Our aim is to optimise the management of diabetic patients with microalbuminuria in our hospital.

\footnotetext{
Proposal for Welsh Diabetic Kidney Disease Cohort Alhadj Ali $M,{ }^{1,2}$ McLaughlin $L^{1}{ }^{1}$ Noyes $J,{ }^{3}$ Fraser $D^{1}$

Wales Kidney Research Unit, Cardiff University School of Medicine, Cardiff

Diabetes Research Group, Cardiff University School of Medicine, Cardiff

Bangor University, Bangor
}

Introduction: Diabetic kidney disease (DKD) is identified as one of the most serious challenges facing the NHS in Wales and the UK. It is a common and feared complication of diabetes mellitus. DKD carries an increased risk of all-cause and cardiovascular mortality such that overall life expectancy is worse than that of people diagnosed with common cancers including lung, breast and prostate. DKD is also the commonest cause of kidney failure in the UK, and the cost to patients and to healthcare providers of DKD is staggering.

Aims and objectives: (1) To set up a new cohort of patients with DKD in Wales for biomarker discovery and validation. (2) To explore how best to further optimise procedures and recruitment by gaining a better understanding of how initial processes are working and to ascertain the experiences and thought processes of patients, clinicians and researchers with respect to study participation and consent to kidney biopsy.

Methods: 100 patients with DKD will be recruited from secondary care diabetes and nephrology services to establish an initial cohort. Patients will undergo detailed clinical evaluation alone $(n=80)$ or with kidney biopsy $(n=20)$, with selection of study arm by patient choice. Interviews will be conducted with 40 cohort participants (20 kidney biopsy and 20 non-biopsy) at cohort entry and at follow-up visit (when kidney biopsy and other results are fed back), with an emphasis on recruitment and consent to kidney biopsy.

\section{"To Biopsy or Not": a rare cause of renal failure Chok Y, Baburaj R, Goel S \\ The Hillingdon Hospitals NHS Foundation Trust}

A 75-year-old man with type 2 diabetes mellitus presented with a 4-week history of abdominal pain and $10 \mathrm{~kg}$ weight loss over 6 months. Physical examination was unremarkable. Blood investigations revealed acute kidney injury. CT abdomen showed a focal plaque of abnormal soft tissue in the left pelvic sidewall encasing the left common iliac vessels and bifurcation, measuring approximately $4.5 \times 1.5 \mathrm{~cm}$, causing obstruction of the left kidney. The patient underwent left ureteric stenting.

Because of the proximity of the pelvic mass to vascular structures and concerns about tumour seeding, it was deemed not amenable to subcutaneous image-guided biopsy. The patient subsequently underwent an open biopsy at the Royal Marsden Hospital. The histology of the biopsy raised a strong suspicion of IgG4-related sclerosing disease (IgG4-RD). The lgG4 level was raised, further supporting the diagnosis of IgG4-RD.

High-dose steroids were started and the patient responded very well with resolution of the pelvis mass on CT 5 months after starting steroids. Glycaemic control was a problem when the patient was put on high-dose steroids and insulin was required during steroid titration.

Discussion: IgG4-RD can present as a retroperitoneal mass which can cause obstructive uropathy. It is important to biopsy the mass as IgG4 in lymph nodes can be non-specific. Awareness of the presentations of lgG4-RD needs to be heightened as it is an entity that responds very well to steroids. Surveillance of other organs for manifestations of IgG4-RD is important as IgG4-RD can present in multiple organs. 